\title{
Positive solutions for generalized Caputo fractional differential equations using lower and upper solutions method
}

\author{
HANAN A. WAHASH ${ }^{a}, *$ (1) ,SATISH K. PANChal ${ }^{a}$ (1) \\ ${ }^{a}$ Department of Mathematics, Dr. Babasaheb Ambedkar Marathwada University, \\ Aurangabad, India.
}

- Received: 03.11.2020 • Accepted: 25.12.2020 • Published Online:30.12.2020

\begin{abstract}
The existence and uniqueness of positive solutions are investigated for a new class of boundary value problems for a fractional differential equation involving generalized Caputo fractional derivative of order $\vartheta(1<\vartheta \leqslant 2)$. Our approach relies on the properties of a green function, Banach's contraction principle, and Schauder's fixed point technique on a cone. Moreover, we use building the upper and lower control functions for analysis of the results of our suggested problem. In the end, two examples are given to justify our acquired results.
\end{abstract}

Keywords: $\sigma$-Caputo fractional differential equations, positive solutions, upper and lower solutions, fixed point theorem.

2010 MSC: 34A08, 34B15,, 34B18, 47H10.

\section{Introduction}

Theory of fractional differential equations (FDEs) has been of considerable interest lately. This is due to the widespread evolution of the fractional calculus (FC) and its applications. FDEs arise in dynamical processes, rheology, fluid flows, viscoelasticity, chemical physics, electrical networks, and numerous other offshoots of science and engineer, e.g., see [1, 2, 3, 4, 5, 6, 7]. As referred to in [8], there have arisen numerous works, in which fractional derivatives (FDs) are utilized for a superior depiction of considered about material properties, mathematical modeling dependent on improved rheological models normally prompts FDEs and to the needed of the formulation of initial conditions to such problems.

Applied problems require definitions of FDs permitting the usage of truly interpretable initial conditions. It should be noticed that most articles and monographs on

*Corresponding author: hawahash86@gmail.com

(C) 2020 SABA. All Rights Reserved. 
FC are committed to the solvability of linear initial FDEs regarding special functions. See $[9,10,11,12,13,14]$.

As of late, there have been some recent papers managing the existence and uniqueness of solutions (or positive solutions) of FDEs by the usage of various methods of nonlinear analysis, see [15, 16, 17, 18, 19, 20, 21, 22, 23, 24, 25, 26, 27]. For instance, the authors in [18] studied the existence and uniqueness of positive solution for the following problem

$$
\left\{\begin{array}{l}
D_{0^{+}}^{\vartheta} \varkappa(\zeta)=f(\zeta, \varkappa(\zeta)), \quad \zeta \in(0,1) \\
\varkappa(0)=0
\end{array}\right.
$$

where $0<\vartheta<1, D_{0^{+}}^{\vartheta}$ is the Riemann-Liouville $\mathrm{FD}$, and $f:[0,1] \times \mathbb{R}^{+} \rightarrow \mathbb{R}^{+}$is continuous function. Wahash et.al., in [25] discussed the existence and uniqueness of a positive solution for the following problem

$$
\left\{\begin{array}{c}
{ }^{c} D_{0^{+}}^{\vartheta, \sigma} \varkappa(\zeta)=f(\zeta, \varkappa(\zeta)), \quad \zeta \in[0,1] \\
\varkappa(0)=\lambda \int_{0}^{1} g(s) \varkappa(s) d s+d,
\end{array}\right.
$$

where $0<\vartheta<1, \lambda \geqslant 0, \mathrm{~d} \in \mathbb{R}^{+},{ }^{\mathrm{C}} \mathrm{D}_{0^{+}}^{\vartheta, \sigma}$ is the generalized Caputo FD of order $\vartheta$ introduced by Almeida [28], $f:[0,1] \times \mathbb{R}^{+} \rightarrow \mathbb{R}^{+}$is continuous function, and $g \in$ $\mathrm{L}^{1}\left([0,1], \mathbb{R}^{+}\right)$.

Motivated by the aforementioned papers and inspired by [28], we investigate the existence and uniqueness of positive solution of the following $\sigma$-Caputo FDFs

$$
\left\{\begin{array}{c}
{ }^{C} D_{0+}^{\vartheta, \sigma} \varkappa(\zeta)+f(\zeta, \varkappa(\zeta))=0, \quad \zeta \in(0,1), \\
\varkappa(0)=m, \varkappa(1)=n,
\end{array}\right.
$$

where $1<\vartheta \leqslant 2, m, n \in \mathbb{R}^{+}(n>m),{ }^{C} D_{0+}^{\vartheta, \sigma}$ is the generalized Caputo FD of order $\vartheta$, $f:[0,1] \times \mathbb{R}^{+} \rightarrow \mathbb{R}^{+}$, and $\sigma:[0,1] \rightarrow \mathbb{R}^{+}$such that $\sigma \in \mathrm{C}^{2}[0,1]$ an increasing function with $\sigma^{\prime}(\zeta) \neq 0$, for all $\zeta \in[0,1]$.

The proposed problem (1.1) is more general than those found in literature, indeed, when we take certain functions of $\sigma$, our problem is reduced to many problems in the frame of classical FDs. Also, the existence of positive solution obtained through constructing the lower and upper control functions of the nonlinear terms without any monotone requirement. Besides, our analysis of the results was limited to the minimum assumptions.

The paper is marshaled as follows: In Section 2, we supply some preludial results. In Section 3, we derive the corresponding fractional integral (FI) equation of (1.1) then we also prove the existence and uniqueness of positive solution by using the upper and lower solution method along with fixed point theorems. Two examples are given in Section 4 . We end the paper with the conclusions section.

\section{Preliminary results}

In this portion, we collect some basic definitions and lemmas related to our results.

Let $\mathrm{C}[0,1]$ be the Banach space with the norm $\|\varkappa\|=\max \{|\varkappa(\zeta)| ; \zeta \in[0,1]\}$ and define the cone 


$$
\mathrm{K}=\{\varkappa \in \mathrm{C}[0,1]: \varkappa(\zeta) \geqslant 0, \quad \zeta \in[0,1]\} .
$$

Let $1<\vartheta \leqslant 2, \varpi:[0,1] \rightarrow \mathbb{R}^{+}$be an integrable function and $\sigma \in \mathrm{C}^{\mathfrak{n}}[0,1]$ an increasing function such that $\sigma^{\prime}(\zeta) \neq 0$, for all $\zeta \in[0,1]$.

Definition 2.1. [1] The $\sigma$-Riemann-Liouville FI of $\varpi$, of order $\vartheta$, is given by

$$
\mathrm{I}_{0+}^{\vartheta, \sigma} \varpi(\zeta)=\frac{1}{\Gamma(\vartheta)} \int_{0}^{\zeta} \sigma^{\prime}(\mathrm{s})(\sigma(\zeta)-\sigma(\mathrm{s}))^{\vartheta-1} \varpi(\mathrm{s}) \mathrm{ds} .
$$

Definition 2.2. [1] The $\sigma$-Riemann-Liouville FD of $\bowtie$, of order $\vartheta>0$ is given by

$$
\mathrm{D}_{0+}^{\vartheta, \sigma} \varpi(\zeta)=\left[\frac{1}{\sigma^{\prime}(\zeta)} \frac{\mathrm{d}}{\mathrm{d} \zeta}\right]^{\mathrm{n}} \mathrm{I}_{0+}^{\mathrm{n}-\vartheta, \sigma} \varpi(\zeta),
$$

where $n=[\vartheta]+1$.

Definition 2.3. [28] The $\sigma$-Caputo FD of $\varpi$, of order $\vartheta$, is given by

$$
{ }^{\mathrm{C}} \mathrm{D}_{0+}^{\vartheta, \sigma} \varpi(\zeta)=\mathrm{D}_{0+}^{\vartheta, \sigma}\left[\varpi(\zeta)-\sum_{\mathrm{k}=0}^{\mathrm{n}-1} \frac{\varpi_{\sigma}^{[\mathrm{k}]}(0)}{\mathrm{k} !}(\sigma(\zeta)-\sigma(0))^{\mathrm{k}}\right] .
$$

where $\mathrm{n}=[\vartheta]+1$, and $\varpi_{\sigma}^{[k]}(\zeta)=\left[\frac{1}{\sigma^{\prime}(\zeta)} \frac{\mathrm{d} \zeta}{\mathrm{d} \zeta}\right]^{\mathrm{k}} \varpi(\zeta)$. For $\vartheta \notin \mathbb{N}$, then

$$
{ }^{C} D_{a+}^{\vartheta, \sigma} \varpi(\zeta)=I_{a+}^{n-\vartheta, \sigma}\left[\frac{1}{\sigma^{\prime}(\zeta)} \frac{d}{d \zeta}\right]^{n} \varpi(\zeta) .
$$

while if $\vartheta=n \in \mathbb{N}$, then ${ }^{C} D_{0+}^{\vartheta, \sigma} \varpi(\zeta)=\varpi_{\sigma}^{[k]}(\zeta)$.

Definition 2.4. A function $\varkappa \in \mathrm{C}[0,1]$ is called a positive solution of the problem (1.1) if $\varkappa(\zeta) \geqslant 0$ for all $\zeta \in[0,1]$ and $\varkappa$ satisfies the problem (1.1).

Lemma 2.5. [28] Let $\vartheta>0$ and $\varpi:[0,1] \rightarrow \mathbb{R}$. Then we have

1) If $\varpi \in C[0,1]$ then ${ }^{C} D_{0+}^{\vartheta, \sigma} I_{0+}^{\vartheta, \sigma} \varpi(\zeta)=\varpi(\zeta)$.

2) If $\varpi \in \mathrm{C}^{n-1}[0,1]$ then $\mathrm{I}_{0+}^{\vartheta, \sigma C} \mathrm{D}_{0+}^{\vartheta, \sigma} \varpi(\zeta)=\varpi(\zeta)-\sum_{k=0}^{n-1} \frac{\varpi_{\sigma}^{[k]}(0)}{k !}[\sigma(\zeta)-\sigma(0)]^{k}$.

Lemma 2.6. $[28,1]$ Let $\vartheta, \xi>0$ and $\varpi:[0,1] \rightarrow \mathbb{R}$. Then

1) $\mathrm{I}_{0+}^{\vartheta, \sigma}[\sigma(\zeta)-\sigma(0)]^{\xi-1}=\frac{\Gamma(\xi)}{\Gamma(\vartheta+\xi)}[\sigma(\zeta)-\sigma(0)]^{\vartheta+\xi-1}$.

2) ${ }^{C} D_{0+}^{\vartheta, \sigma}[\sigma(\zeta)-\sigma(0)]^{\xi-1}=\frac{\Gamma(\xi)}{\Gamma(\xi-\vartheta)}[\sigma(\zeta)-\sigma(0)]^{\xi-\vartheta-1}$.

3) ${ }^{\mathrm{C}} \mathrm{D}_{0+}^{\vartheta, \sigma}[\sigma(\zeta)-\sigma(0)]^{\mathrm{k}}=0, \quad \forall \mathrm{k} \in(0,1, \ldots, \mathrm{n}-1), \mathrm{n} \in \mathbb{N}$.

4) $\mathrm{I}_{0+}^{\vartheta, \sigma} \mathrm{I}_{0+}^{\xi, \sigma} \varpi(\zeta)=\mathrm{I}_{0+}^{\vartheta+\xi, \sigma} \mathrm{f}(\zeta)$. 
Lemma 2.7. [28] Let $\vartheta>0$ and $\varpi:[0,1] \rightarrow \mathbb{R}$. Then $\mathrm{I}_{0+}^{\vartheta, \sigma}$ is bounded from $\mathrm{C}[0,1]$ into $\mathrm{C}[0,1]$. Moreover,

$$
\mathrm{I}_{0+}^{\vartheta, \sigma} \varpi(0)=\lim _{\zeta \rightarrow 0^{+}} \mathrm{I}_{0+}^{\vartheta, \sigma} \varpi(\zeta)=0 .
$$

Theorem 2.8. [29] Let $\Phi$ be a Banach space with a contraction mapping $Q: \Phi \rightarrow \Phi$. Then, $Q$ has a unique fixed-point $\varkappa$ in $\Phi$.

Theorem 2.9. [29] Let $\Phi$ be a Banach space and let $S$ a convex, closed, bounded subset of $\Phi$. If $\mathrm{Q}: \mathrm{S} \longrightarrow \mathrm{S}$ is a continuous map such that the set $\{\mathrm{Q} \varkappa: \varkappa \in \mathrm{S}\}$ is relatively compact in $\Phi$. Then $\mathrm{Q}$ has at least one fixed point.

\section{Main results}

In this portion, we prove the existence and uniqueness of positive solutions for (1.1). Before starting, we introduce the following lemma:

Lemma 3.1. Let $1<\vartheta \leqslant 2$ and $\varpi:[0,1] \rightarrow \mathbb{R}^{+}$is a continuous function. Then the linear $\sigma$ Caputo FDFs

$$
\begin{aligned}
{ }^{\mathrm{C}} \mathrm{D}_{0+}^{\vartheta, \sigma} \varkappa(\zeta)+\varpi(\zeta) & =0, \quad 0<\zeta \leqslant 1, \\
\varkappa(0)=\mathrm{m}, \varkappa(1) & =\mathrm{n},
\end{aligned}
$$

is equivalent to

$$
\varkappa(\zeta)=m+(n-m) \Upsilon(\zeta)+\int_{0}^{1} \sigma^{\prime}(s) G_{\sigma}(\zeta, s) \varpi(s) d s
$$

where

$$
\mathrm{G}_{\sigma}(\zeta, \mathrm{s})=\frac{\Upsilon(\zeta)}{\Gamma(\vartheta)}\left\{\begin{array}{cc}
(\sigma(1)-\sigma(\mathrm{s}))^{\vartheta-1}-\frac{1}{\gamma(\zeta)}(\sigma(\zeta)-\sigma(s))^{\vartheta-1}, & 0 \leqslant s \leqslant \zeta \leqslant 1, \\
(\sigma(1)-\sigma(s))^{\vartheta-1}, & 0 \leqslant \zeta \leqslant s \leqslant 1,
\end{array}\right.
$$

with $\Upsilon(\zeta):=\frac{\mathcal{N}(\zeta)}{\mathcal{N}(1)}$, and $\mathcal{N}(\zeta):=\sigma(\zeta)-\sigma(0)$.

Proof. Applying $\mathrm{I}_{0^{+}}^{\vartheta, \sigma}$ on both sides of the first equation of (3.1), and using Lemma 2.5, we reduce the equation ${ }^{C} D_{0+}^{\vartheta, \sigma} \varkappa(\zeta)=\varpi(\zeta)$, into its equivalent FI equation as

$$
\begin{aligned}
\varkappa(\zeta)= & -\mathrm{I}_{0+}^{\vartheta, \sigma} \varpi(\zeta)+\mathrm{c}_{0}+\mathrm{c}_{1}[\sigma(\zeta)-\sigma(0)] \\
= & -\frac{1}{\Gamma(\vartheta)} \int_{0}^{\zeta} \sigma^{\prime}(\mathrm{s})(\sigma(\zeta)-\sigma(\mathrm{s}))^{\vartheta-1} \varpi(\mathrm{s}) \mathrm{d} s \\
& +\mathrm{c}_{0}+\mathrm{c}_{1}(\sigma(\zeta)-\sigma(0)) .
\end{aligned}
$$

Then we have $\varkappa(0)=c_{0}$ and

$$
\varkappa(1)=-\frac{1}{\Gamma(\vartheta)} \int_{0}^{1} \sigma^{\prime}(s)(\sigma(1)-\sigma(s))^{\vartheta-1} \varpi(s) d s+c_{0}+c_{1}(\sigma(1)-\sigma(0)) .
$$


From the boundary conditions $\varkappa(0)=m$ and $\varkappa(1)=n$, we obtain $c_{0}=m$ and

$$
c_{1}=\frac{n-m}{\sigma(1)-\sigma(0)}+\frac{1}{\sigma(1)-\sigma(0)}\left(I_{0+}^{\vartheta, \sigma} \varpi(s)\right)(1) .
$$

Substitute the values of $c_{0}$ and $c_{1}$ in (3.4), we get

$$
\begin{aligned}
\varkappa(\zeta)= & m+(n-m) \Upsilon(\zeta)+\frac{\Upsilon(\zeta)}{\Gamma(\vartheta)} \int_{0}^{1} \sigma^{\prime}(s)(\sigma(1)-\sigma(s))^{\vartheta-1} \varpi(s) d s \\
& -\frac{1}{\Gamma(\vartheta)} \int_{0}^{\zeta} \sigma^{\prime}(s)(\sigma(\zeta)-\sigma(s))^{\vartheta-1} \varpi(s) d s \\
= & m+(n-m) \Upsilon(\zeta)+\frac{\Upsilon(\zeta)}{\Gamma(\vartheta)} \int_{0}^{\zeta} \sigma^{\prime}(s)(\sigma(1)-\sigma(s))^{\vartheta-1} \varpi(s) d s \\
& +\frac{\Upsilon(\zeta)}{\Gamma(\vartheta)} \int_{\zeta}^{1} \sigma^{\prime}(s)(\sigma(1)-\sigma(s))^{\vartheta-1} \varpi(s) d s \\
& -\frac{1}{\Gamma(\vartheta)} \int_{0}^{\zeta} \sigma^{\prime}(s)(\sigma(\zeta)-\sigma(s))^{\vartheta-1} \varpi(s) d s \\
= & m+(n-m) \Upsilon(\zeta)+\frac{\Upsilon(\zeta)}{\Gamma(\vartheta)}\left(\int_{0}^{\zeta} \sigma^{\prime}(s)(\sigma(1)-\sigma(s))^{\vartheta-1} \varpi(s) d s\right. \\
& \left.-\frac{1}{\Upsilon(\zeta)} \sigma^{\prime}(s)(\sigma(\zeta)-\sigma(s))^{\vartheta-1} \varpi(s) d s\right) \\
& +\frac{\Upsilon(\zeta)}{\Gamma(\vartheta)} \int_{\zeta}^{1} \sigma^{\prime}(s)(\sigma(1)-\sigma(s))^{\vartheta-1} \varpi(s) d s \\
= & m+(n-m) \Upsilon(\zeta)+\int_{0}^{1} \sigma^{\prime}(s) G_{\sigma}(\zeta, s) \varpi(s) d s .
\end{aligned}
$$

This shows that (3.2) is satisfied.

As result of Lemma 3.1, we get the following Lemma:

Lemma 3.2. Assume that $f:[0,1] \times \mathbb{R}^{+} \rightarrow \mathbb{R}^{+}$is a continuous, and $\varkappa$ be a function. Then the $\sigma$ - Caputo FDFs (1.1) is equivalent to

$$
\varkappa(\zeta)=m+(n-m) \Upsilon(\zeta)+\int_{0}^{1} \sigma^{\prime}(s) G_{\sigma}(\zeta, s) f(s, \varkappa(s)) d s .
$$

Note that, The equation (3.5) is also equivalent to

$$
(Q \varkappa)(\zeta)=m+(n-m) \Upsilon(\zeta)+\int_{0}^{1} \sigma^{\prime}(s) G_{\sigma}(\zeta, s) f(s, \varkappa(s)) d s,
$$

where $\mathrm{Q}: \mathrm{K} \rightarrow \mathrm{K}$ is an operator such that $\mathrm{Q} \varkappa(\zeta)=\varkappa(\zeta), \varkappa(\zeta) \in \mathrm{C}[0,1]$.

Lemma 3.3. [8] The function $\mathrm{G}_{\sigma}$ defined by (3.3) satisfies

1. $G_{\sigma}(\zeta, s)>0$ for $\zeta, s \in(0,1)$.

2. $\Gamma(\vartheta) \max _{0 \leqslant \zeta \leqslant 1} G_{\sigma}(\zeta, s)=[\sigma(1)-\sigma(s)]^{\vartheta-1}, s \in(0,1)$. 
Definition 3.4. [18] The upper and lower control functions are defined by

$$
\bar{\Delta}(\zeta, \varkappa)=\sup _{a \leqslant \eta \leqslant \varkappa} f(\zeta, \eta) \text { and } \underline{\Delta}(\zeta, \varkappa)=\inf _{\varkappa \leqslant \eta \leqslant b} f(\zeta, \eta) \text {, }
$$

respectively, where $a, b \in \mathbb{R}^{+}(b>a)$ and $\varkappa \in[a, b]$.

It is clear that $\bar{\Delta}(\zeta, \varkappa)$ and $\Delta(\zeta, \varkappa)$ are non-decreasing on $\varkappa$ and

$$
\underline{\Delta}(\zeta, \varkappa) \leqslant f(\zeta, \varkappa) \leqslant \bar{\Delta}(\zeta, \varkappa) .
$$

Definition 3.5. [18] Let $\bar{\varkappa}(\zeta), \underline{\varkappa}(\zeta) \in \mathrm{K}$ and $\mathrm{a} \leqslant \underline{\varkappa}(\zeta) \leqslant \bar{\varkappa}(\zeta) \leqslant \mathrm{b}$ satisfy

$$
\begin{gathered}
{ }^{C} D_{0+}^{\vartheta, \sigma} \bar{\varkappa}(\zeta)+\bar{\Delta}(\zeta, \bar{\varkappa}(\zeta)) \geqslant 0, \quad 0 \leqslant \zeta \leqslant 1, \\
\quad \varkappa(0) \geqslant m, \quad \bar{\varkappa}(1) \geqslant n,
\end{gathered}
$$

or

$$
\bar{\varkappa}(\zeta) \geqslant m+(n-m) \Upsilon(\zeta)+\int_{0}^{1} \sigma^{\prime}(s) G_{\sigma}(\zeta, s) \bar{\Delta}(s, \bar{\varkappa}(s)) d s
$$

and

$$
\begin{aligned}
& { }^{C} D_{0+\underline{\varkappa}}^{\vartheta, \sigma}(\zeta)+\underline{\Delta}(\zeta, \underline{\varkappa}(\zeta)) \leqslant 0, \quad 0 \leqslant \zeta \leqslant 1, \\
& \underline{\varkappa}(0) \leqslant m, \quad \underline{\varkappa}(1) \leqslant n,
\end{aligned}
$$

or

$$
\underline{\varkappa}(\zeta) \leqslant m+(n-m) \Upsilon(\zeta)+\int_{0}^{1} \sigma^{\prime}(s) G_{\sigma}(\zeta, s) \underline{\Delta}(s, \underline{\varkappa}(s)) d s .
$$

Then, $\bar{\varkappa}(\zeta)$ and $\underline{\varkappa}(\zeta)$ are called upper and lower solutions for (1.1).

Now, we give the following hypothese:

$\left(\mathrm{H}_{1}\right) f:[0,1] \times \mathbb{R}^{+} \rightarrow \mathbb{R}^{+}$is continuous.

$\left(\mathrm{H}_{2}\right)$ There exists a constant $\mathrm{L}_{f}>0$ such that

$$
\left|f\left(\zeta, \varkappa_{1}\right)-f\left(\zeta, \varkappa_{2}\right)\right| \leqslant L_{f}\left|\varkappa_{1}-\varkappa_{2}\right|, \forall \zeta \in[0,1], \varkappa_{1}, \varkappa_{2} \in \mathbb{R}^{+}
$$

$\left(\mathrm{H}_{3}\right)$ There exists a constant $\rho>0$ such that

$$
|f(\zeta, \varkappa)| \leqslant \rho, \quad(\zeta, \varkappa) \in[0,1] \times \mathbb{R}^{+} .
$$

Theorem 3.6. Suppose that $\left(\mathrm{H}_{1}\right)-\left(\mathrm{H}_{3}\right)$ hold. Let $\bar{\varkappa}(\zeta), \underline{\varkappa}(\zeta)$ are upper, lower solutions of problem (1.1). Then the $\sigma$-Caputo FDF (1.1) has at least a solution $\varkappa(\zeta)$. Moreover,

$$
\underline{\varkappa}(\zeta) \leqslant \varkappa(\zeta) \leqslant \bar{\varkappa}(\zeta), \quad \zeta \in[0,1]
$$

Proof. Convert the problem (1.1) into a fixed point problem. Consider the operator $\mathrm{Q}: \mathrm{K} \rightarrow \mathrm{K}$ defined by (3.6). Then we shall make use of Theorem 2.9 to verify that $\mathrm{Q}$ has a fixed point. The proof will be presented in some steps.

Step1: The operator $\mathrm{Q}: \mathrm{K} \rightarrow \mathrm{K}$ is compact. 
From the continuity and nonnegativity of $G_{\sigma}(\zeta, s)$ and $f(s, \varkappa(s))$, the operator $Q$ is continuous. Define a ball

$$
\mathcal{B}_{\mathrm{r}}=\{\varkappa \in \mathrm{K}:\|\varkappa\| \leqslant \mathrm{r}, \quad \zeta \in[0,1]\},
$$

Let $\varkappa \in \mathcal{B}_{\mathrm{r}}$ and by assumptions $\left(\mathrm{H}_{1}\right)$ and $\left(\mathrm{H}_{3}\right)$. Then we get

$$
\begin{aligned}
|(Q \varkappa)(\zeta)| & \leqslant m+(n-m)|\Upsilon(\zeta)|+\int_{0}^{1} \sigma^{\prime}(s) G_{\sigma}(\zeta, s)|f(s, \varkappa(s))| d s \\
& \leqslant m+(n-m)|\Upsilon(\zeta)|+\rho \max _{0 \leqslant \zeta \leqslant 1} \int_{0}^{1} \sigma^{\prime}(s) G_{\sigma}(\zeta, s) d s \\
& =m+(n-m)\left|\frac{\sigma(\zeta)-\sigma(0)}{\sigma(1)-\sigma(0)}\right|+\frac{\rho(\sigma(1)-\sigma(0))}{\Gamma(\vartheta+1)} \\
& \leqslant n+\frac{\rho(\sigma(1)-\sigma(0))^{\vartheta}}{\Gamma(\vartheta+1)} .
\end{aligned}
$$

Hence

$$
\|(\mathrm{Q} \varkappa)\| \leqslant \mathrm{n}+\frac{\rho(\sigma(1)-\sigma(0))^{\vartheta}}{\Gamma(\vartheta+1)} .
$$

This show that $\mathrm{Q}: \mathcal{B}_{\mathrm{r}} \rightarrow \mathcal{B}_{\mathrm{r}}$ is uniformly bounded.

Now, we prove that $Q$ is equicontinuous. For each $\varkappa \in \mathcal{B}_{\mathrm{r}}$. Then for $\zeta_{1}, \zeta_{2} \in[0,1]$ with $\zeta_{1}<\zeta_{2}$, we have

$$
\begin{aligned}
\left|(Q \varkappa)\left(\zeta_{2}\right)-(Q \varkappa)\left(\zeta_{1}\right)\right| \leqslant & (n-m)\left|\Upsilon\left(\zeta_{2}\right)-\Upsilon\left(\zeta_{1}\right)\right| \\
& +\int_{0}^{1} \sigma^{\prime}(s)\left|G_{\sigma}\left(\zeta_{2}, s\right)-G_{\sigma}\left(\zeta_{1}, s\right)\right||f(s, \varkappa(s))| d s .
\end{aligned}
$$

Now, we need to simplify the expression $\left|G_{\sigma}\left(\zeta_{2}, s\right)-G_{\sigma}\left(\zeta_{1}, s\right)\right|$. Let us consider for $0 \leqslant s \leqslant \zeta \leqslant 1$,

$$
\begin{aligned}
& \left|G_{\sigma}\left(\zeta_{2}, s\right)-G_{\sigma}\left(\zeta_{1}, s\right)\right| \\
= & \mid \frac{\Upsilon\left(\zeta_{2}\right)}{\Gamma(\vartheta)}(\sigma(1)-\sigma(s))^{\vartheta-1}-\frac{1}{\Upsilon\left(\zeta_{2}\right)}\left(\sigma\left(\zeta_{2}\right)-\sigma(s)\right)^{\vartheta-1} \\
& -\frac{\Upsilon\left(\zeta_{1}\right)}{\Gamma(\vartheta)}(\sigma(1)-\sigma(s))^{\vartheta-1}+\frac{1}{\Upsilon\left(\zeta_{1}\right)}\left(\sigma\left(\zeta_{1}\right)-\sigma(s)\right)^{\vartheta-1} \mid \\
= & \mid \frac{(\sigma(1)-\sigma(s))^{\vartheta-1}}{(\sigma(1)-\sigma(0)) \Gamma(\vartheta)}\left(\left(\sigma\left(\zeta_{2}\right)-\sigma(0)\right)-\left(\sigma\left(\zeta_{1}\right)-\sigma(0)\right)\right) \\
& +\frac{1}{\Gamma(\vartheta)}\left(\left(\sigma\left(\zeta_{1}\right)-\sigma(s)\right)^{\vartheta-1}-\left(\sigma\left(\zeta_{2}\right)-\sigma(s)\right)^{\vartheta-1}\right) \mid
\end{aligned}
$$


Let $g_{1}(\zeta)=\sigma(\zeta)-\sigma(0)$ and $g_{2}(\zeta)=(\sigma(\zeta)-\sigma(s))^{\vartheta-1}$. By applying the mean value theorem, we obtain

$$
\begin{aligned}
& \left|G_{\sigma}\left(\zeta_{2}, s\right)-G_{\sigma}\left(\zeta_{1}, s\right)\right| \\
= & \left|\zeta_{2}-\zeta_{1}\right|\left(\frac{(\sigma(1)-\sigma(s))}{(\sigma(1)-\sigma(0)) \Gamma(\vartheta)} g_{1}^{\prime}\left(\xi_{1}\right)+\frac{1}{\Gamma(\vartheta)} g_{2}^{\prime}\left(\xi_{2}\right)\right)
\end{aligned}
$$

where $\xi_{1}, \xi_{2} \in\left(\zeta_{1}, \zeta_{2}\right)$. Therefore, as $\zeta_{2}-\zeta_{1} \rightarrow 0,\left|(\mathrm{Q} \varkappa)\left(\zeta_{2}\right)-(\mathrm{Q} \varkappa)\left(\zeta_{1}\right)\right| \rightarrow 0$, which means that $\left(\mathrm{Q} \mathcal{B}_{\mathrm{r}}\right)$ is equicontinuous. Hence by the Arzela-Ascoli theorem, $\mathrm{Q}: \mathcal{B}_{\mathrm{r}} \rightarrow \mathcal{B}_{\mathrm{r}}$ is completely continuous.

Step2: To apply Theorem 2.9 , we need to prove $\mathrm{Q}: \mathcal{S} \rightarrow \mathcal{S}$, where

$$
\mathcal{S}=\{\varkappa(\zeta): \varkappa(\zeta) \in K, \underline{\varkappa}(\zeta) \leqslant \varkappa(\zeta) \leqslant \bar{\varkappa}(\zeta), \zeta \in[0,1]\}
$$

From (3.8), it follows that $\mathcal{S}$ is a closed, convex, and bounded subset of $C\left([0,1], \mathbb{R}^{+}\right)$. For

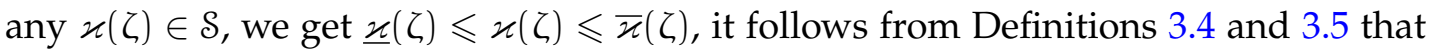

$$
\begin{aligned}
(\mathrm{Q} \varkappa)(\zeta) & =\mathrm{m}+(\mathrm{n}-\mathrm{m}) \Upsilon(\zeta)+\int_{0}^{1} \sigma^{\prime}(\mathrm{s}) \mathrm{G}_{\sigma}(\zeta, \mathrm{s}) \mathrm{f}(\mathrm{s}, \varkappa(\mathrm{s})) \mathrm{ds} \\
& \leqslant \mathrm{m}+(\mathrm{n}-\mathrm{m}) \Upsilon(\zeta)+\int_{0}^{1} \sigma^{\prime}(\mathrm{s}) \mathrm{G}_{\sigma}(\zeta, \mathrm{s}) \bar{\Delta}(\mathrm{s}, \bar{\varkappa}(\mathrm{s})) \mathrm{d} s \\
& \leqslant \bar{\varkappa}(\zeta),
\end{aligned}
$$

and

$$
\begin{aligned}
(\mathrm{Q} \varkappa)(\zeta) & =\mathrm{m}+(\mathrm{n}-\mathrm{m}) \Upsilon(\zeta)+\int_{0}^{1} \sigma^{\prime}(\mathrm{s}) \mathrm{G}_{\sigma}(\zeta, \mathrm{s}) \mathrm{f}(\mathrm{s}, \varkappa(\mathrm{s})) \mathrm{d} s \\
& \geqslant \mathrm{~m}+(\mathrm{n}-\mathrm{m}) \Upsilon(\zeta)+\int_{0}^{1} \sigma^{\prime}(\mathrm{s}) \mathrm{G}_{\sigma}(\zeta, \mathrm{s}) \underline{\Delta}(\mathrm{s}, \underline{\varkappa}(\mathrm{s})) \mathrm{d} \mathrm{s} \\
& \geqslant \underline{\varkappa}(\zeta) .
\end{aligned}
$$

So, $\underline{\varkappa}(\zeta) \leqslant \mathrm{Q} \varkappa(\zeta) \leqslant \bar{\varkappa}(\zeta), 0 \leqslant \zeta \leqslant 1$ which gives $\mathrm{Q} \varkappa(\zeta) \in \mathcal{S}, \forall \zeta \in[0,1]$. This shows that $\mathrm{Q}: \mathcal{S} \rightarrow \mathcal{S}$. As an outcome of Theorem 2.9, Q has at least one fixed point $\varkappa(\zeta) \in \mathcal{S}, 0 \leqslant \zeta \leqslant 1$. Thus, the problem (1.1) has at least one solution $\varkappa(\zeta) \in \mathrm{C}[0,1]$ and $\underline{\varkappa}(\zeta) \leqslant \varkappa(\zeta) \leqslant \bar{\varkappa}(\zeta), \zeta \in[0,1]$.

Corollary 3.7. Suppose that $\mathrm{f}:[0,1] \times \mathbb{R}^{+} \rightarrow \mathbb{R}^{+}$is continuous, and there exist $\aleph_{2} \geqslant \aleph_{1}>0$ such that

$$
\aleph_{1} \leqslant f(\zeta, \sigma) \leqslant \aleph_{2}, \quad(\zeta, \sigma) \in[0,1] \times \mathbb{R}^{+} .
$$

Then there exists at least a solution $\varkappa(\zeta)$ of the Caputo-FDF (1.1). Moreover,

$$
\underline{\varkappa}(\zeta) \leqslant \varkappa(\zeta) \leqslant \bar{\varkappa}(\zeta), \text { for } \zeta \in[0,1]
$$

where

$$
\bar{\varkappa}(\zeta)=m+(n-m) \Upsilon(\zeta)+\left((\mathcal{N}(1))^{\vartheta-1}-(\mathcal{N}(\zeta))^{\vartheta-1}\right) \frac{\aleph_{2}}{\Gamma(\vartheta+1)} \mathcal{N}(\zeta),
$$




$$
\underline{\varkappa}(\zeta)=m+(n-m) \Upsilon(\zeta)+\left((\mathcal{N}(1))^{\vartheta-1}-(\mathcal{N}(\zeta))^{\vartheta-1}\right) \frac{\aleph_{1}}{\Gamma(\vartheta+1)} \mathcal{N}(\zeta) .
$$

Proof. From Definition 3.4 and hypothesis (3.9), we have

$$
\aleph_{1} \leqslant \Delta(\zeta, \sigma) \leqslant \bar{\Delta}(\zeta, \sigma) \leqslant \aleph_{2}, \quad(\zeta, \sigma) \in[0,1] \times[a, b] .
$$

Now, we consider the following $\sigma$-Caputo problem

$$
\begin{gathered}
{ }^{\mathrm{C}} \mathrm{D}_{0+}^{\vartheta, \sigma} \bar{\varkappa}(\zeta)+\mathrm{\aleph}_{2}=0, \quad 0 \leqslant \zeta \leqslant 1, \\
\bar{\varkappa}(0)=\mathrm{m}, \quad \bar{\varkappa}(1)=\mathrm{n} .
\end{gathered}
$$

Duo to Lemma 3.1, the $\sigma$-Caputo problem (3.12) has a positive solution

$$
\begin{aligned}
\bar{\varkappa}(\zeta)= & m+(n-m) \Upsilon(\zeta)+\frac{\aleph_{2} \curlyvee(\zeta)}{\Gamma(\vartheta)} \int_{0}^{1} \sigma^{\prime}(s)(\sigma(1)-\sigma(s))^{\vartheta-1} \mathrm{ds} \\
& -\frac{\aleph_{2}}{\Gamma(\vartheta)} \int_{0}^{\zeta} \sigma^{\prime}(\mathrm{s})(\sigma(\zeta)-\sigma(s))^{\vartheta-1} \mathrm{ds} \\
= & \mathrm{m}+(\mathrm{n}-\mathrm{m}) \Upsilon(\zeta)+\frac{\aleph_{2} \curlyvee(\zeta)}{\Gamma(\vartheta+1)}(\sigma(1)-\sigma(0))^{\vartheta} \\
& -\frac{\aleph_{2}}{\Gamma(\vartheta+1)}(\sigma(\zeta)-\sigma(0))^{\vartheta} \\
= & m+(\mathrm{n}-\mathrm{m}) \Upsilon(\zeta)+\frac{\aleph_{2}}{\Gamma(\vartheta+1)} \frac{(\sigma(\zeta)-\sigma(0))}{(\sigma(1)-\sigma(0))}(\sigma(1)-\sigma(0))^{\vartheta} \\
& -\frac{\aleph_{2}}{\Gamma(\vartheta+1)}(\sigma(\zeta)-\sigma(0))^{\vartheta} \\
= & m+(\mathrm{n}-\mathrm{m}) \Upsilon(\zeta)+\left((\mathcal{N}(1))^{\vartheta-1}-(\mathcal{N}(\zeta))^{\vartheta-1}\right) \frac{\aleph_{2}}{\Gamma(\vartheta+1)} \mathcal{N}(\zeta) .
\end{aligned}
$$

By (3.11), we conclude that

$$
\bar{\varkappa}(\zeta) \geqslant m+(n-m) \Upsilon(\zeta)+\int_{0}^{1} \sigma^{\prime}(s) G_{\sigma}(\zeta, s) \bar{\Delta}(s, \bar{\varkappa}(s)) d s .
$$

Thus, the function $\bar{\varkappa}(\zeta)$ is the upper solution of the $\sigma$-Caputo FDE (1.1). In the above same way, if the $\sigma$-Caputo problem of the type

$$
\begin{gathered}
{ }^{\mathrm{C}} \mathrm{D}_{0+\underline{\varkappa}}^{\vartheta, \sigma}(\zeta)+\aleph_{1}=0, \quad 0 \leqslant \zeta \leqslant 1, \\
\underline{\varkappa}(0)=\mathrm{m}, \quad \underline{\varkappa}(1)=\mathrm{n} .
\end{gathered}
$$

Obviously, the $\sigma$-Caputo problem (3.13) has also a positive solution

$$
\begin{aligned}
\underline{x}(\zeta)= & m+(n-m) \Upsilon(\zeta)+\frac{\aleph_{1} \curlyvee(\zeta)}{\Gamma(\vartheta)} \int_{0}^{1} \sigma^{\prime}(s)(\sigma(1)-\sigma(s))^{\vartheta-1} \mathrm{ds} \\
& -\frac{\aleph_{1}}{\Gamma(\vartheta)} \int_{0}^{\zeta} \sigma^{\prime}(s)(\sigma(\zeta)-\sigma(s))^{\vartheta-1} \mathrm{ds} \\
= & m+(n-m) \Upsilon(\zeta)+\left((\mathcal{N}(1))^{\vartheta-1}-(\mathcal{N}(\zeta))^{\vartheta-1}\right) \frac{\aleph_{1}}{\Gamma(\vartheta+1)} \mathcal{N}(\zeta) .
\end{aligned}
$$


By (3.11), we conclude that

$$
\underline{\varkappa}(\zeta) \leqslant m+(n-m) \Upsilon(\zeta)+\int_{0}^{1} \sigma^{\prime}(s) G_{\sigma}(\zeta, s) \underline{\Delta}(s, \underline{\varkappa}(s)) d s .
$$

Thus, the function $\underline{\varkappa}(\zeta)$ is the lower solution of the $\sigma$-Caputo FDE (1.1).

By Theorem (3.6), we get that the $\sigma$-Caputo FDE (1.1) has at least one positive solution $\varkappa(\zeta) \in \mathcal{S}$, which justifies the inequalitiy (3.10).

The final result is based on Theorem 2.8 .

Theorem 3.8. Suppose that $\left(\mathrm{H}_{1}\right)-\left(\mathrm{H}_{2}\right)$ hold. If $\frac{(\sigma(1)-\sigma(0))^{\vartheta}}{\Gamma(\vartheta+1)} \mathrm{L}_{\mathrm{f}}<1$, Then the $\sigma$-Caputo FDE (1.1) has a unique positive solution $\varkappa(\zeta) \in \mathrm{C}[0,1]$.

Proof. Theorem 3.6 shows that the $\sigma$-Caputo FDE (1.1) has at least one positive solution in $\mathrm{K}$. Now we consider $\mathrm{Q}: \mathrm{K} \rightarrow \mathrm{K}$ defined by (3.6). Hence, we need only to show that $\mathrm{Q}$ is contraction map in $\mathrm{C}[0,1]$. Let $\varkappa_{1}, \varkappa_{2} \in \mathrm{C}[0,1]$ and $\zeta \in[0,1]$. Then we have

$$
\begin{aligned}
& \left\|\mathrm{Q} \varkappa_{1}-\mathrm{Q} \varkappa_{2}\right\| \\
= & \max _{\zeta \in[0,1]}\left|\left(\mathrm{Q} \varkappa_{1}\right)(\zeta)-\left(\mathrm{Q} \varkappa_{2}\right)(\zeta)\right| \\
\leqslant & \max _{\zeta \in[0,1]} \int_{0}^{1} \sigma^{\prime}(\mathrm{s}) \mathrm{G}_{\sigma}(\zeta, s)\left|\mathrm{f}\left(\mathrm{s}, \varkappa_{1}(\mathrm{~s})\right)-\mathrm{f}\left(\mathrm{s}, \varkappa_{2}(\mathrm{~s})\right)\right| \mathrm{d} s \\
\leqslant & \frac{1}{\Gamma(\vartheta)} \int_{0}^{1} \sigma^{\prime}(\mathrm{s})(\sigma(1)-\sigma(\mathrm{s}))^{\vartheta-1} \mathrm{~L}_{\mathrm{f}}\left\|\varkappa_{1}-\varkappa_{2}\right\| \mathrm{d} s \\
\leqslant & \frac{(\sigma(1)-\sigma(0))^{\vartheta}}{\Gamma(\vartheta+1)} \mathrm{L}_{\mathrm{f}}\left\|\varkappa_{1}-\varkappa_{2}\right\| .
\end{aligned}
$$

Since $\frac{(\sigma(1)-\sigma(0))^{\vartheta}}{\Gamma(\vartheta+1)} \mathrm{L}_{\mathrm{f}}<1, \mathrm{Q}$ is contraction mapping. As a result of Theorem 2.8, we can infer that $\mathrm{Q}$ has a unique fixed point which is the unique positive solution of (1.1) on $[0,1]$.

\section{Examples}

In this portion, we provide two examples to enlighten our results.

Example 4.1. Consider the following $\sigma$-Caputo FDE

$$
\begin{gathered}
{ }^{\mathrm{C}} \mathrm{D}_{0^{+}}^{\frac{3}{2} ; e^{\zeta}} \varkappa(\zeta)=1+\frac{\varkappa(\zeta)}{37+\sin (\varkappa(\zeta))}, \quad 0 \leqslant \zeta \leqslant 1, \\
\varkappa(0)=1, \quad \varkappa(1)=2,
\end{gathered}
$$

where $\vartheta=\frac{3}{2}, \sigma(\zeta)=e^{\zeta}, \mathrm{m}=1, \mathrm{n}=2$, and $\mathrm{f}(\zeta, \varkappa)=1+\frac{\varkappa}{37+\sin (\varkappa)}$. It is not difficult to see that $\mathrm{f}$ is a continuous and nonnegative function. For $\zeta \in[0,1]$ and $\varkappa, v \in[0, \infty)$, we get $|f(\zeta, \varkappa)-f(\zeta, v)| \leqslant \frac{1}{37}|x-y|$. Here $L_{f}=\frac{1}{37}$. Therefore $\left(H_{1}\right)$ and $\left(H_{2}\right)$ are satisfied. 
Moreover, by some simple calculations, we get $\frac{(\sigma(1)-\sigma(0))^{\vartheta}}{\Gamma(\vartheta+1)} \mathrm{L}_{\mathrm{f}} \approx 0.05<1$. All assumptions of Theorem 3.8 hold. Therefore, Theorem 3.8 assurances that (4.1) has a unique positive solution $\varkappa(\zeta) \in \mathrm{C}[0,1]$.

Example 4.2. Consider the following $\sigma$-Caputo FDE

$$
\begin{gathered}
{ }^{\mathrm{C}} \mathrm{D}_{0^{+}}^{\frac{3}{2} ; e^{\frac{\zeta}{2}}} \varkappa(\zeta)=\frac{1}{\zeta+6}\left(\frac{\zeta \varkappa(\zeta)}{1+\varkappa(\zeta)}+6\right), 0 \leqslant \zeta \leqslant 1, \\
\varkappa(0)=1, \quad \varkappa(1)=2,
\end{gathered}
$$

Note that, $\vartheta=\frac{3}{2}, \sigma(\zeta)=e^{\frac{\zeta}{2}}, m=1, n=2$, and $f(\zeta, \varkappa)=\frac{1}{\zeta+6}\left(\frac{\zeta \varkappa}{1+\varkappa}+6\right)$. Since $f$ is continuous and

$$
\frac{6}{7} \leqslant f(\zeta, \varkappa) \leqslant 1
$$

for all $(\zeta, \varkappa) \in[0,1] \times \mathbb{R}^{+}$. Thus, $\aleph_{1}=\frac{6}{7}$ and $\aleph_{2}=1$. Hence by Corollary 3.7, the $\sigma$-Caputo FDE (4.2) has a positive solution which verifies $\underline{\varkappa}(\zeta) \leqslant \varkappa(\zeta) \leqslant \bar{\varkappa}(\zeta)$ where

$$
\begin{aligned}
& \bar{\varkappa}(\zeta)=1+\frac{\sqrt{e^{\zeta}}-1}{\sqrt{e}-1}\left(\sqrt{e^{\frac{1}{2}}-1}-\sqrt{e^{\frac{\zeta}{2}}-1}\right) \frac{\sqrt{e^{\frac{\zeta}{2}}-1}}{\Gamma\left(\frac{5}{2}\right)}, \\
& \varkappa(\zeta)=1+\frac{\sqrt{e^{\zeta}}-1}{\sqrt{e}-1}\left(\sqrt{e^{\frac{1}{2}}-1}-\sqrt{e^{\frac{\zeta}{2}}-1}\right) \frac{6 \sqrt{e^{\frac{\zeta}{2}}-1}}{7 \Gamma\left(\frac{5}{2}\right)},
\end{aligned}
$$

are respectively the upper and lower solutions of $\sigma$-Caputo FDE (4.2). Furthermore, for all $(\zeta, \varkappa) \in[0,1] \times \mathbb{R}^{+},|f(\zeta, \varkappa)| \leqslant 1=\rho$. Thus, since all the hypotheses in Theorem 3.6 and Corollary 3.7 are fulfilled, our results can be applied to the $\sigma$-Caputo FDE.

\section{Conclusions}

We have deliberated a category of BVPs for nonlinear FDEs involving $\sigma$-Caputo fractional derivative. With the Green function, the fixed point techniques of Banach and Schauder, and the upper and lower solutions method, we have proven the existence and uniqueness of positive solutions for the suggested problem. In the end, two examples to justify the main results. Many results of the corresponding problems that hold standard fractional operators are covered as special cases of (1.1). The announced results here are a new and significant contribution to the current literature on the theme.

\section{References}

[1] Kilbas AA, Srivastava MH and Trujillo JJ (2006). "Theory and Applications of Fractional Differential Equations". North-Holland Mathematics studies 204, Elsevier Science, Amsterdam.

[2] Oldham KB and Spanier J (1974). "The Fractional Calculus". Academic Press, New York and London.

[3] Ross B (1975). "The Fractional Calculus and its Applications". Lecture Notes in Mathematics 475, Springer-Verlag, Berlin.

[4] Nonnenmacher TF and Metzler R (1995). On the Riemann-Liouville fractional calculus and some recent applications. Fractals 3: 557566. 
[5] Tatom FB (1995). The relationship between fractional calculus and fractals. Fractals 3: 217-229.

[6] Podlubny I (1999). "Fractional Differential Equations". Mathematics in Science and Engineering 198, Academic Press, New york, London, Toronto.

[7] Samko SG, Kilbas AA and Marichev OI (1993). "Fractional Integral and Derivatives: Theory and Applications". Gordon and Breach, Switzerland.

[8] Wahash HA and Panchal SK. (2020). Positive solutions for generalized two-term fractional differential equations with integral boundary conditions. J. Math. Anal. Modeling 1(1): 47-63. 10011024.https://doi.org/10.48185/jmam.v1i1.35

[9] Khaldi R, Guezane-Lakoud A (2017). Upper and Lower Solutions Method for Higher Order Boundary Value Problems. Progress Frac. Diff. Applic. 3: 53-57. https://doi.org/10.1080/1726037X.2017.1327162

[10] Jaikumar S and Palani P (2016). Applications of fractional differential equations. Int. J. Sci. Tech. Hum. (IJSTH), 3 (2).

[11] Constantinescu CD, Ramirez JM and Zhu WR (2019). An application of fractional differential equations to risk theory. Finance Stoch. 23: 1001-1024.https://doi.org/10.1007/s00780-019-00400-8

[12] Valério D, Trujillo JJ, Rivero M, Machado JT and Baleanu D (2013). Fractional calculus: A survey of useful formulas. Eur. Phys. J. Spec. Top. 222(8): 1827-1846. https://doi.org/10.1140/epjst/e2013-01967-y

[13] Mainardi F (1997). "Fractional calculus, fractals and fractional calculus in continuum mechanics". Springer-Verlag, Wien.

[14] Hilfer R (2000). "Applications of fractional calculus in physics". Singapore: World scientific 35(12):87130.

[15] Ardjouni A and Djoudi A (2019). Existence and uniqueness of positive solutions for first-order nonlinear Liouville-Caputo fractional differential equations. Sao Paulo J. Math. Sci. http://doi.org/10.1007/s40863019-00147-2

[16] Li H, Liu L and Wu Y (2015). Positive solution for singular nonlinear fractional differential equation with integral boundary conditions. Bound. Value Prob. 232 https://doi.org/10.1186/s13661-015-0493-3

[17] Abdo MS, Wahash HA and Panchal SK (2018). Positive solution of a fractional differential equation with integral boundary conditions. J. Appl. Math. Comput. Mech. 17(3): 5-15. https:/ / doi.org/10.17512/jamcm.2018.3.01 DOI

[18] Li N, Wang C (2013). New existence results of positive solution for a class of nonlinear fractional differential equations. Acta Mathematica Scientia 33B: 847-854. https://doi.org/10.1016/S0252-9602(13)60044-2

[19] Rahimy M (2010). Applications of fractional differential equations. Appl. Math. Sci. 4(50): 2453-2461.

[20] Wang G, Liu S, Agrawal RP and Zhang L (2013). Positive Solution of Integral Boundary Value Problem Involving Riemann-Liouville Fractional Derivative. J. Frac. Calc. Appl. 4(2): 312-321.

[21] Sun Y, Zhao M (2014). Positive solutions for a class of fractional differential equations with integral boundary conditions. Appl. Math. Lett. 34: 17-21. https://doi.org/10.1016/j.aml.2014.03.008

[22] Wang Y, Liu L and Wu Y (2011). Positive solutions for a nonlocal fractional differential equation. Nonlinear Anal. 74: 3599-3605. https://doi.org/10.1016/j.na.2011.02.043

[23] Abdo MS (2020). Further results on the existence of solutions for generalized fractional quadratic functional integral equations. J. Math. Anal. Modeling 11: 33-46. https://doi.org/10.48185/jmam.v1i1.2

[24] Abdo MS, Abdeljawad T, Ali SM, Shah K and Jarad F (2020). Existence of positive solutions for weighted fractional order differential equations. Chaos Solitons Fractals 141, 110341. https:/ / doi.org/10.1016/j.chaos.2020.110341

[25] Wahash HA, Panchal SK and Abdo MS (2020). Positive solutions for generalized Caputo fractional differential equations with integral boundary conditions. J. Mathematical Modeling 8(4): 393-414. https:/ / doi.org/10.22124/JMM.2020.16125.1407

[26] Afshari H, Abdo MS and Alzabut J (2020). Further results on existence of positive solutions of generalized fractional boundary value problems. Adv. Differ. Equ. 2020: 600. https://doi.org/10.1186/s13662-02003065-2

[27] Abdo MS, Jeelani M, Saeed MA and Shah K (2020). Positive solutions for fractional boundary value problems under a generalized fractional operator. Authorea Preprints. https://doi.org/10.22541/au.159196543.33370456

[28] Almeida R (2017). A Caputo fractional derivative of a function with respect to another function. Commun. Nonlinear Sci. 44: 460-481. https:/ / doi.org/10.1016/j.cnsns.2016.09.006

[29] Zhou Y (2014). "Basic theory of fractional differential equations". Singapore: World Scientific. 6. 